\title{
Aplicabilidad de las teorías de comunicación en salud en el campo de la educación nutricional
}

\section{Applicability of health communication theories in nutrition education}

\section{RESUMEN}

La solución de los problemas de salud relacionados con nutrición requiere el diseño, ejecución y evaluación de intervenciones efectivas que incrementen el bienestar de la población. El objetivo fue analizar la aplicabilidad de las teorías existentes de comunicación en salud para el campo de la educación nutricional. Se recopilaron y analizaron las teorías de comunicación y modelos de comportamiento en salud utilizados con mayor frecuencia para la promoción de la salud. La modificación de comportamientos relacionados con alimentación y nutrición, que conduzca a hábitos alimentarios saludables requiere de intervenciones planeadas teniendo en cuenta las características socioeconómicas, culturales, experiencias alimentarias previas, actitudes, creencias, percepciones, motivaciones, normas culturales, redes sociales y familiares y condiciones ambientales.

Palabras clave: Educación alimentaria y nutricional; Comunicación; Educación; Promoción de la salud.

\section{INTRODUCCIÓN}

La educación nutricional es un eje transversal, esencial para todas las áreas de la nutrición, ya sea clínica, de servicios de alimentos, ciencia de los alimentos o nutrición pública. Si bien tradicionalmente se ha entendido que el fin de la educación alimentaria y nutricional es la transmisión de conocimientos, el verdadero propósito subyace en la necesidad de aportar elementos científicos y técnicos que promuevan y permitan mantener estados adecuados de salud integral y favorecer cambios de comportamientos, como la elección de alimentos saludables que, consecuentemente, contribuyen a la prevención de enfermedades.

El papel de la educación nutricional es fundamental para identificar las necesidades educacionales sobre alimentación, mediante el diagnóstico de conocimientos en salud de los colectivos y su relación con la alimentación. Además, la educación nutricional permite considerar las necesidades, motivaciones, creencias, actitudes, intereses, normas sociales y culturales que rigen las prácticas alimentarias, favoreciendo la orientación de estrategias para la solución de problemas de salud.

La educación junto a la comunicación para la salud,

\author{
Edna Gamboa-Delgado', Ericka Escalante Izeta², \\ Alejandra Amaya-Castellanos ${ }^{3}$.
}

1. Escuela de Nutrición y Dietética. Universidad Industrial de Santander. Bucaramanga, Colombia. 2. Universidad Iberoamericana. Ciudad de México, México. 3. Instituto Nacional de Salud Pública de México. Cuernavaca, México.
Este trabajo fue recibido el 15 de marzo de 2017. Aceptado con modificaciones: 21 de julio de 2017. Aceptado para ser publicado: 22 de agosto de 2017.

se constituyen en estrategias de acción fundamentales para el logro del mejoramiento de las condiciones nutricionales de las poblaciones. La comunicación, particularmente, además de cumplir con el objetivo de mantener informada a la población acerca de asuntos relacionados con la nutrición, se fundamenta esencialmente en las interacciones y mediaciones que se dan a través de ella, y esto es lo que le da relevancia al proceso de entender y comprender la relación existente entre la nutrición y la salud. Bajo esta línea, aparecen cada vez con mayor frecuencia investigaciones, programas e intervenciones con enfoques más holísticos 
y menos limitados para el abordaje de la educación nutricional. La mayoría de ellos se dan a la luz de las teorías del comportamiento, con las que buscan comprender las razones por las que los individuos pueden o no modificar fácilmente comportamientos relacionados con la alimentación y poder transformar actitudes frente a hábitos alimenticios puntuales. Así mismo, muchos de estos programas se fundamentan en modelos de intervención procedentes del estudio científico del proceso de comunicación humana (audiencia, mensaje, fuente, canal y efecto del mensaje). Existen propuestas pedagógicas e investigaciones interesantes de nivel individual, interpersonal y medio que intentan integrar de manera efectiva la educación y la comunicación para avanzar en la modificación de comportamientos. Unas se valen de las ventajas que ofrecen las tecnologías de la información y la comunicación (TIC) para ampliar los espacios virtuales de encuentro con las audiencias, diseñar mensajes a la medida o aplicar instrumentos que pueden predecir la etapa de cambio en la que se ubican las personas; en tanto que otras apuestan a la participación para generar procesos de comunicación horizontales en los que se compartan saberes y se centran en fortalecer y desarrollar habilidades que hagan posibles cambios permanentes. No obstante, el reto sigue siendo conseguir que la audiencia pase de la decisión a la acción y mantenga los cambios en el tiempo. El objetivo de este ensayo fue analizar intervenciones bajo modelos y teorías existentes de comunicación en salud y su aplicabilidad en el campo de la educación nutricional.

\section{Metodología}

La búsqueda de los artículos se realizó a través de Google Schoolar, usando las bases de datos de PubMed, Scielo, Redalyc, Science Direct y Elsevier. Como criterio de selección se consideraron aquellos artículos publicados entre 2000 y 2017. Los términos de búsqueda fueron: Health Communication and Nutrition Education Interventions, Health Communication and Nutrition Education Programs. Fueron tomados en cuenta artículos en español e inglés. Teorías de Comunicación en Salud.

Existen diversas teorías de comunicación en salud que pueden ser usadas en la enseñanza de la nutrición. Resulta necesario comprender sus diferentes aproximaciones a la construcción de conocimientos con la finalidad de elegir la más conveniente de acuerdo con las características de la audiencia objetivo. Habrá que decir que la comunicación, ya sea interpersonal, comunitaria o masiva y la articulación entre ellas, puede contribuir no sólo a la diseminación de información y generación de conocimientos, sino al entendimiento de determinados comportamientos y su consecuente modificación. Algunos referentes teóricos de la comunicación, provienen del estudio científico del proceso de comunicación humana y han sido empleados a través de los años, como la teoría crítica de la escuela de Frankfurt, que refiere a la comunicación dentro de un amplio contexto social y cuestiona el papel de los medios de comunicación sobre el poder político y económico'. Esta teoría invita a la reflexión acerca del rol tan influyente de los medios en el refuerzo de ideologías dominantes en la opinión pública. Otra teoría utilizada es la de percepción del peligro, la cual conecta salud y enfermedad bajo una relación simple de causa-efecto ${ }^{2}$ que puede resultar reduccionista dada la multi-causalidad y complejidad de las enfermedades y que no siempre es motivo suficiente para cambiar las prácticas o comportamientos considerados nocivos para la salud. Otra teoría es la de Mercadeo social, consistente en la aplicación de los principios del mercadeo comercial al análisis, planeación, ejecución y evaluación de programas diseñados para influir en los comportamientos de poblaciones con el fin de mejorar su bienestar ${ }^{3}$. Esta teoría ha sido usada en la promoción de la salud, para divulgación y reforzamiento de comportamientos saludables. Así mismo, lo ha hecho la Comunicación persuasiva ${ }^{4}$, la cual se enfoca en diez pasos específicos que una persona, expuesta a un mensaje, da para adoptar finalmente un comportamiento. La eficacia del mensaje se atribuye, en gran medida, a quien lo emite (persuasor) y a su habilidad para engranar con delicadeza y destacar con ingenio cinco elementos que el receptor (persuadido) valorará: a) credibilidad de la fuente; b) diseño del mensaje (claro y contundente); c) canal de comunicación; d) conocimiento del grupo objetivo y e) descripción del comportamiento promovido.Entre tanto, en el modelo de Comunicación para el cambio de comportamiento ${ }^{5}$, enlazado al anterior, se elaboran mensajes que persuaden a las personas para que se apropien de conductas que beneficien su salud, intentando neutralizar la inquietud y el miedo que genera la enfermedad. De igual forma lo hace la teoría de la difusión de innovaciones ${ }^{6}$ en la que la transferencia de nuevos conocimientos permite a las personas y a su comunidad mejorar las condiciones de vida y salud. El carácter de "nuevo/novedoso" del comportamiento promovido es justo lo que permite su diseminación en la audiencia. En este sentido, los medios masivos son un canal eficaz para difundir la información y motivar la toma de decisiones, pero es realmente a través del uso del canal interpersonal cuando se activan los mecanismos de adopción de la nueva práctica.Para ello, la audiencia debe transitar por cinco estadios que implican: percibir que el nuevo comportamiento es mejor que el que se va a modificar (Ventaja relativa); verificar que concuerda con los valores, costumbres y necesidades de su comunidad (Compatibilidad); comprender a cabalidad en qué consiste (Complejidad); experimentar con él antes de adherirlo a su vida (Posibilidad de poner a prueba) y ver los resultados concretos de dicho cambio entre sus pares (Observar/evaluar). Por último, están la Comunicación para el desarrollo ${ }^{7,8}$ y la Comunicación participativa o comunitaria ${ }^{9}$, las cuales centran su interés, y hacen hincapié, en la horizontalidad del acto comunicativo y en compartir saberes entre interlocutores, más que en la producción de mensajes per se (en esta también se inscribe el modelo de comunicación para el cambio social ${ }^{10,11}$. En ellas, la participación es considerada un componente indispensable 
en los procesos de comunicación y desarrollo, promoviendo procesos colectivos de aprendizaje que propician el diálogo, el pensamiento crítico, el desarrollo de habilidades y, a la larga, el empoderamiento de las personas. Lo interesante es que dichos procesos suelen estar vinculados a intervenciones de largo aliento que se articulan con programas y políticas públicas de carácter social.

\section{Aplicación de las teorías al campo de la Nutrición}

La cultura alimentaria es un constructo social que contiene símbolos y significados que determinan la aceptación, preferencia, cohesión grupal y consumo de alimentos ${ }^{12}$. De manera paralela, los objetivos de la educación alimentaria y nutricional se basan en preservar los alimentos y formas de preparación tradicionales de alto valor nutritivo, contribuir al control de enfermedades relacionadas con la dieta y favorecer la equidad en cuanto al beneficio de una alimentación que cubra las necesidades biológicas, sociales y culturales de la población. Por tanto, bajo el escenario de la alimentación como un proceso de aprendizaje sociocultural, la aplicabilidad de las teorías de comunicación en salud es de total relevancia. Considerar el conocimiento existente sobre estas teorías sirve de base para el diseño de políticas y programas, con componentes educativos, que aporten soluciones viables a las problemáticas alimentarias.

El incremento de las enfermedades crónicas en todo el mundo, especialmente las relacionadas con nutrición como diabetes, obesidad, cáncer y enfermedades cardiovasculares, reflejan las interacciones existentes entre ambiente, comportamientos personales y biología. En temas específicos referidos a la salud nutricional se ha aplicado la comunicación persuasiva, a través de intervenciones a medida en la internet ${ }^{13-15}$ y de campañas de comunicación mediadas sobre cambios de comportamiento para la prevención de enfermedades cardiovasculares ${ }^{16}$, mientras que el modelo de difusión de innovaciones se ha empleado para fomentar el uso de vitaminas entre la población $n^{17,18}$. Por tanto, se reconoce cada vez más la importancia de la educación nutricional como eje esencial de la promoción de la salud y prevención de la enfermedad.

Evidencia de diversos estudios ha mostrado que algunos programas que incluyen componentes de educación nutricional han tenido impacto positivo en desenlaces relacionados con la nutrición. Las intervenciones educativas suelen incluir desde contenidos en el plan curricular, políticas en los servicios de alimentación escolar, juegos educativos, marketing social, y consejería individual, hasta la adopción de nuevas tecnologías, como el uso de telefonía celular ${ }^{19}$ y aplicaciones para el envío de mensajes nutricionales y para el monitoreo de la adherencia a planes nutricionales $\mathrm{s}^{20-22}$. En la internet también se han generado estrategias que emplean mensajes hechos a medida de la audiencia empleando mecanismos de personalización, retroalimentación y adaptación tomados del mercadeo social que incluyen panfletos y medios interactivos, que han conseguido importantes modificaciones en prácticas de nutrición, actividad física y pérdida de peso entre los participantes, logrando mantener cambios por más de un año ${ }^{23}$. Esas intervenciones de educación nutricional pueden complementarse con acciones de actividad física, entrega de transferencias monetarias o de transferencias en especie. Los desenlaces para los cuales estas intervenciones educativas han resultado efectivas son, en su mayoría, reducción de ganancia excesiva de peso, mayor consumo de frutas y verduras ${ }^{22}$, aumento de la duración de lactancia materna exclusiva ${ }^{24}$, disminución en la prevalencia de desnutrición crónica en niños ${ }^{25}$ e incremento en peso y talla infanti ${ }^{26,27}$, entre otras. La evidencia señala que estos programas son más efectivos cuando la educación se basa en el comportamiento/acción, más que solo en el conocimiento y cuando se conecta la teoría, la investigación y la práctica ${ }^{28}$. Adicionalmente, suele lograrse mayor efectividad cuando las intervenciones educativas tienen una duración mayor a un año ${ }^{23}$, involucran diversos actores como padres de familia, maestros, alumnos, pareja y sectores como sociedad civil, organismos gubernamentales y no gubernamentales, industria de alimentos, y academia. Para ello, algunas intervenciones han elaborado mensajes empleando la comunicación para el cambio de comportamiento por medio de cual motivan la adopción y sostenimiento de un hábito o estilo de vida específico y para elaborar herramientas que ayuden en la planeación de programas de intervención familiar, en las que hay un interés especial por la participación comunitaria ${ }^{29}$ o por generar el debate social ${ }^{30}$. Aunque los cambios de comportamiento no son fáciles de lograr, los resultados esperados pueden obtenerse bajo condiciones de cambio en la postura de los profesionales de salud, al dejar de lado la aproximación pedagógica tradicional (autoritaria y vertical) y pasar a estrategias activas y críticas en niveles horizontales de empatía y comprensión con la audiencia objetivo de los mensajes nutricionales. Tanto en los niveles interpersonal, grupal o comunitario se ha evidenciado un aumento en las investigaciones que abordan el estudio de la audiencia y el diseño de mensaje con el propósito de mejorar su penetración en la audiencia objetivo ${ }^{31}$. La radio local, por ejemplo, se ha empleado para la transmisión de dichos mensajes creados a partir de la persuasión. Se ha integrado el componente de participación al proceso de desarrollo, grabación y evaluación de narrativas de salud (radio dramas) en el que miembros de la comunidad han sido capacitados en la creación de mensajes y producción de programas radiales, con la intención de contribuir a la construcción de la capacidad comunitaria para que sean ellas quienes creen sus propias intervenciones en salud ${ }^{32}$ a través de la comunicación participativa ${ }^{33}$. Si la educación nutricional se realiza abordando componentes totalmente acordes al contexto social y cultural de la población de referencia, además de elementos motivacionales de participación y desarrollo, se puede lograr el cambio de actitudes y creencias a través de estrategias de comunicación efectivas. Es así como, para intentar reducir la pobreza extrema en contextos rurales y urbanos, se han puesto en marcha iniciativas de seguridad 
alimentaria y nutricional que consideran estrategias y líneas de acción que tienen como ejes transversales la equidad de género y la comunicación para el desarrollo ${ }^{34}$. La literatura refiere que para conseguir la modificación de actitudes y creencias también es necesario incluir un componente de acción que puede ayudar a facilitar la intención de cambio de comportamiento, una vez se fijen metas y se logren habilidades de autorregulación cognitiva.

Finalmente, el desarrollo de componentes ambientales que promuevan la educación alimentaria y nutricional, considerando las características del entorno y las políticas públicas que lo sustentan, permitirá que los educadores nutricionales trabajen de manera activa junto con tomadores de decisiones política para promover las condiciones ambientales que favorezcan la acción.

\section{CONCLUSIONES}

Este ensayo incita a la reflexión sobre la necesidad de considerar las teorías de comunicación en salud en el diseño de programas de educación nutricional, con la intención de modificar hábitos y prácticas alimentarias aprendidas, heredadas y adquiridas en el contexto cultural, social, económico e histórico de cada persona.

Quedan preguntas para la reflexión como ¿cuál teoría de comunicación en salud es la que puede generar mayor efectividad sostenible en términos de desenlaces relacionados con nutrición? o ¿cuáles son los supuestos bajos los cuales dichas teorías pueden funcionar mejor?

El ideal de la educación nutricional es que contribuya a planear, implementar y evaluar intervenciones efectivas en beneficio de la salud de individuos y colectivos. Resulta claro que solo proporcionar información no es suficiente, se requiere generar cambios de actitud hacia la alimentación y de comportamientos en cuanto a estilos de vida.Para construir conocimientos alimentarios que se traduzcan, de manera efectiva, en formas de alimentación saludables, se requiere tener en cuenta el simbolismo alimentario construido socioculturalmente, el poder adquisitivo, la disponibilidad de alimentos, los conocimientos científicos relacionados con la nutrición y la alimentación, la influencia de los medios de información masiva, el entorno y las modas alimentarias.

Es recomendable la planeación de intervenciones educativas, que tomen como base los problemas reales y las experiencias de las comunidades, además de elegir adecuadamente las teorías educativas o conductuales que las sustenten, ya que algunas pueden tener escaso significado para determinado contexto cultural y no ser aplicables a quienes va dirigida. La evaluación de proceso e impacto, así como los resultados de intervenciones de educación nutricional efectivas son de alta implicación para los formuladores de políticas en la medida que se logre la reducción de problemas de salud relacionados con la nutrición. Así mismo, resulta necesario seguir investigando con mayor profundidad sobre las estrategias pedagógicas adecuadas que favorezcan la adopción de nuevos comportamientos en salud, integradas de manera ecológica con la oferta de alimentos, las políticas públicas y los entornos sociales y construidos que favorezcan la prevención de enfermedades. De esta manera se pueden trabajar conjuntamente los aspectos individuales que condicionan la elección de alimentos, con los elementos estructurales que en gran medida determinan las condiciones de salud-enfermedad-atención de los colectivos.

\section{BIBLIOGRAFÍA}

1. Sierra F. Teoría crítica y comunicología. El legado de la escuela de Frankfurt. Constelaciones. Rev Teoría Crítica 2011; 3: 349356.

2. García del Castillo JA. Concepto de percepción de riesgo y su repercusión en las adicciones. Health and Addictions 2012; 12(2): 133-151.

3. Grimaldi D. Marketing social para la promoción de la salud. Gran Canaria: Universidad de las Palmas. 1996.

4. McGuire W. Theoretical foundations of campaigns. En: Rice RE, Paysley WJ, editors. Public communication campaigns. Beverly Hills: Sage Publications; 1981. p. 41-70.

5. Smith, WA et al. A World Against Aids: Communication for Behavior Change. Washington, DC: Academy for Educational Development 1993.

6. Rogers E. Diffusion of innovations. New York: The Free Press; 1995.

7. Waisbord S. Family Tree of Theories, Methodologies and Strategies in Development Communication: Convergences and Differences. In: The Communication Initiative Network. 2001 [citado 19 ago 2017]. Disponible en: www.comminit. $\mathrm{com} / \mathrm{global} / \mathrm{content} /$ family-tree-theories-methodologies-andstrate gies-development-communication-convergences

8. Beltrán L. La comunicación para el desarrollo en Latinoamérica: Un reencuentro de medio siglo. Rev Anagramas 2006; 4(8): 53-76.

9. Anyaegbunam, C. Mefalopulos, P. and Moetsabi, T. Facilitating Grassroots Participation in Development: New Training Models and Techniques. In S.A. White (Ed). The Art of Facilitaing Participation. New Delhi, India: SAGE Publications 1999. $p$ 207-228.

10. Organización de las Naciones Unidas para la Agricultura y la Alimentación. Oficina Regional FAO para América Latina y el Caribe. Comunicación para el cambio social; 2003.

11. Gumucio-Dragón, A. El cuarto mosquetero: La comunicación para el cambio social. Invest y Desarrollo 2004; 12(1): 2-23.

12. Díaz MC. Preferencias alimentarias como alternativa al estudio de patrón dietético. Rev Esp Nutr Comunitaria 2002; 8(1-2): 29-34.

13. Oenema A, Brug J, Lechner L. Web-based taylored nutrition education: results of a randomized controlled trial. Health Educ Res 2001; 16: 647-660.

14. Bensley R, Mercer N, Brusk J, Underhile R, Rivas J, Anderson I, Kelleher D, Lupella M, Jager A. The eHealth Behavior Management Model: A Stage-based Approach to Behavior Change and Management. Prev Chronic Dis 2004; 1(4): 3-13.

15. Hackman C, Knowlden A. Theory of reasoned action and theory of planned behavior-based dietary interventions in adolescents and young adults: a systematic review. Adoles Health Med Ther 2014; 5: 101-114.

16. Punam K, Lehmann D. Designing Effective Health Communications: A Meta-analysis. JPP\&M 2008; 27(2): 117130.

17. Barker K. Diffusion of innovations: a world tour. J Health 
Commun 2004; 9: 131-137.

18. Diez $E$, Juárez $O$, Villamarín F. Intervenciones de promoción de la salud basada en modelos teóricos. Med Clin. 2005; 125(5): 193-197.

19. Delisle C, Sandin S, Forsum E, Henriksson H, Trolle-Lagerros $Y$, Larsson C. A web and mobile phone-based intervention to prevent obesity in 4-year-olds (MINISTOP): A populationbased randomized controlled trial. BMC Public Health 2015; 15: 95.

20. Silveira JAC, Taddei JA, Guerra PH, Nobre MR. The effect of participation in school-based nutrition education interventions on body mass index: A meta-analysis of randomized controlled community trials. Preven Med 2013; 56: 237-243.

21. Guerra PH, Nobre MR, Silveira JÁ, Aguiar JA. School based physical activity and nutritional education interventions on body mass index. A metaanalysis of randomised community trials Project PANE. Preven Med 2014; 61: 81-89.

22. Turner, $T$, Spruijt-Metz D, Wen CKF, Hingle MD. Prevention and treatment of pediatric obesity using mobile and wireless technologies: a systematic review. Pediatr Obes 2015; 10(6): 403-409.23. Enwald K, Huotari A. Preventing the Obesity Epidemic by Second Generation Tailored Health Communication: An Interdisciplinary Review. J Med Internet Res 2010; 12(2): e24.

24. Bhutta ZA, Ahmed T, Black RE, Cousens S, Dewey K, Giugliani $E$, et al. What works? Interventions for maternal and child undernutrition and survival. Lancet 2008; 371(9610): 417-440.

25. Penny ME, Creed-Kanashiro HM, Robert RC, Narro MR, Caulfield $L E$, Black RE. Effectiveness of an educational intervention delivered through the health services to improve nutrition in young children: a cluster-randomized controlled trial. Lancet 2005; 365: 1863-1872.
26. Santos I, Victora CG, Martines J, et al. Nutrition counseling increases weight gain among Brazilian children. I Nutr 2001; 131: 2866-2873.

27. Imdad A, Yakoob MY, BhuttaZA.Impact of maternal education about complementary feeding and provision of complementary foods on child growth in developing countries. BMC Public Health 2011; 11 (Supp/ 3): S25.

28. Contento IR. Nutrition education: linking research, theory, and practice. Asia Pac J Clin Nut 2008; 17(1): 176-179.

29. Salem RM, Berstein J, Sullivan TM. Tools for Behavior Change Communication. INFO Project. 2008 [citado 19 ago 2017]; 16:2-8. Disponible en: https//:www.k4health.org/sites/default/ files/BCCTTools.pdf30. Mohammed S. Personal communication networks and the effects of entertainment-edication radio soap opera in Tanzania. J Health Commun 2001; 6(2): 137-154.

31. Kim JN, Park SC, Yoo SW, Shen H. Mapping Health Communication Scholarship: Breadth, Depth, and Agenda of Published Research in Health Communication. Health Commun 2010; 25(6): 487-503.

32. Racicot-Matta C, Wilked M, Egeland G. Development of radio dramas for health communication pilot intervention in Canadian Inuit communities. Health Promot Int 2004; 31(1): 175-186.

33. Fraser C, Restrepo-Estrada S. Community radio Handbook, Paris: UNESCO. 2000.

34. Organización de las Naciones Unidas para la Alimentación y la Agricultura. Sistematización de la experiencia en San juan de Limay. Modelo de intervención en Seguridad alimentaria y nutricional a nivel municipal [Internet]. Primera edición. Managua: FAO; 2012. [citado 19 ago 2017]. Disponible en: https://coin.fao.org/coinstatic/cms/media/11/13523266649840/ limay_sistematizacion_20-03-2012.pdf 